\title{
Comunicación
}

\section{PSEUDOPREÑEZ EN CABRAS LECHERAS - ESTUDIO DE CASO}

\author{
Pseudopregnancy in Dairy Goats - A Case Study
}

Edgar Villanueva R. ${ }^{1}$, Irma Celi M. ${ }^{2}$, Alfredo Delgado C. ${ }^{3}$

\section{RESUMEN}

El objetivo del presente estudio fue determinar la presencia de hidrometra o pseudopreñez en 38 cabras lecheras criadas bajo un régimen de explotación intensiva. El diagnóstico se realizó mediante ultrasonografía transabdominal a los 45 días del servicio por monta natural. Se consideró una cabra pseudogestante cuando se observó una imagen anecogénica, con la sola presencia de fluido y trabéculas de paredes finas. La incidencia de la hidrometra fue de 5.3\%. Los animales afectados fueron tratados con prostaglandina comercial $\left(\mathrm{PGF}_{2 \alpha}\right)$, corrigiéndose la anomalía y presentando celo dentro de los 4 días del tratamiento. Una cabra quedó gestante y la otra volvió a presentar la hidrometra.

Palabras clave: hidrometra, cabra, ultrasonido

\section{AbSTRACT}

The aim of this study was to determine the presence of hydrometra or pseudopregnancy in 38 dairy goats raised under intensive system. The diagnosis was done using transabdominal ultrasonography at 45 days of natural service. The affected goats were diagnosed as positive when the uterus presented a visible accumulation of fluid and presence of trabeculae in the image. The incidence of a hydrometra was of $5.3 \%$. Both affected animals were successfully treated with commercial prostaglandin ( $\left.\mathrm{PGF}_{2 \alpha}\right)$, and estruses occurred 4 days after the treatment. One goat got pregnant while the hydrometra recurred in the other goat.

Key words: hydrometra, goat, ultrasound

\footnotetext{
${ }^{1}$ Aldea Ecológica Instituto Ecológico para el Desarrollo. Lima, Perú. E-mail: edran99@hotmail.com ${ }^{2}$ Departamento de Ciencias Agroforestales, Universidad de Huelva, España

${ }^{3}$ Clínica de Animales Mayores, Facultad de Medicina Veterinaria, Universidad Nacional Mayor de San Marcos, Lima, Perú. E-mail: aldelgadoc@gmail.com
} 
La crianza de caprinos en el Perú es una actividad ganadera que aún es considerada de forma secundaria y que mayormente se encuentra en manos de personas con bajos recursos técnicos. El sistema de crianza realizado a nivel de costa central es del tipo rastrojero, donde los animales aprovechan los residuos de cosechas y la vegetación circundante de campos de cultivo y acequias. A pesar de esto, la producción caprina ha aumentado en los últimos años, sobre todo por la mayor aceptación de productos derivados de leche de cabra en los mercados de la capital; lo cual está teniendo un impacto favorable en el desarrollo económico y social en la población rural (Arroyo, 1998).

En este aspecto, cobra importancia el manejo productivo y de salud de los rebaños, con el fin de asegurar una producción de leche continua durante todo el año. Sin embargo, esto es difícil de realizar debido al manejo empírico de los animales y a la falta de instalaciones apropiadas. A menudo, aparecen problemas reproductivos en los rebaños, que en la mayoría de los casos no son tomados en cuenta por el desconocimiento de los productores y por la escasa información técnica que se dispone en el país para esta especie (Batista et al., 2001).

La hidrometra o pseudopreñez es un estado patológico que se presenta con frecuencia en la especie caprina, comprometiendo la vida productiva del animal dentro del hato (Salles y Araujo, 2008). Está descrita como la acumulación de fluido aséptico dentro del útero y con la manifestación clínica similar a una cabra gestante (ausencia de celos, reducción de la producción láctea y aumento del volumen abdominal), asociado además, a la presencia de un cuerpo lúteo funcional y elevados niveles de progesterona sérica (Madrid-Bury y González-Stagnaro, 1995). Su diagnóstico en el caprino es relativamente fácil de realizar mediante un examen ecográfico, especialmente entre los 40 y 70 días de la supuesta gestación (Hesselink, 1993).
La incidencia de la pseudopreñez es muy variable (de 3 a $21 \%$, con una media de $9 \%$ ), pudiendo presentarse con más frecuencia en rebaños con elevada producción láctea y cuando se realizan tratamientos hormonales para sincronización de celos (Hesselink, 1993). Este reporte presenta la incidencia, diagnóstico y tratamiento de la pseudopreñez en la especie caprina en base a ecografías de rutina realizadas en cabras lecheras.

La población en estudio estuvo compuesta por 38 cabras (33 Saanen, 4 Alpina y 1 criolla), perteneciente al Instituto Ecológico para el Desarrollo (Aldea Ecológica), localizada en el distrito de Carabayllo, Lima. El sistema de explotación era del tipo intensivo y con una alimentación en base a heno de alfalfa, concentrado comercial y orujo de cebada. El apareamiento se realizaba por monta natural, empleando de manera cotidiana dos machos Saanen y un Alpino, los cuales no presentaban anormalidades reproductivas aparentes.

El diagnóstico de gestación se realizó por ultrasonografía transabdominal, empleando un ecógrafo portátil Chison 600 Vet, con transductor de tipo convexo multifrecuencia de 2.5 a $5.0 \mathrm{Mhz}$, siguiendo el procedimiento sugerido por Pieterse y Taverne (1986) para ecografías en tiempo real de cabras gestantes. Los animales contaban, aproximadamente, con 45 días de gestación al momento de la evaluación ecográfica. Se consideró una cabra pseudogestante cuando se observó una imagen anecogénica, con la sola presencia de fluido y trabéculas de paredes finas (Fig. 1), a diferencia de una cabra preñada que se identificó por la presencia de placentomas y, en algunos casos, del feto (Fig. 2).

De las 38 cabras diagnosticadas, 32 (84.2\%) se encontraron preñadas y 2 (5.3\%) mostraron evidencia ecográfica de pseudopreñez (Cuadro 1). A pesar que en la granja sólo se utiliza monta natural, se observó la ocurrencia de este problema reproductivo en dos hembras, resultado se- 
Cuadro 1. Resultados del diagnóstico por ultrasonografía transabdominal en 38 cabras

\begin{tabular}{lc}
\hline Grupos & Frecuencia (\%) \\
\hline Preñadas & 84.2 \\
Vacías & 10.5 \\
Pseudopreñez & 5.3 \\
\hline Total & 100.0 \\
\hline
\end{tabular}

mejante al 3.1\% reportado en cabras Isla Canaria (Batista et al., 2001) y de 3.4\% en rebaños de cabras criollas venezolanas (Madrid-Bury y González-Stagnaro, 1995).
A las cabras afectadas con pseudopreñez se les administró $0.7 \mathrm{~mL}$ de Tiaprost ( $\mathrm{PGF}_{2 \alpha}$ comercial). Los animales empezaron a eliminar el líquido uterino a las 12 horas del tratamiento, lo cual se prolongó durante cerca de 48 horas. El celo post-tratamiento se presentó al $4^{\circ}$ día, donde volvieron a ser cubiertas. Una de las cabras preñó y tuvo una gestación normal, mientras que la segunda cabra tuvo una recidiva de la patología.

Se puede concluir que la pseudopreñez en la cabra es una alteración de presentación cotidiana, siendo relativamente sencilla de diagnosticar a través de una observación ecográfica; asimismo, se le puede revertir con tratamiento luteolítico a base de PGF $_{2 \alpha}$, permitiendo la recuperación de la actividad ovárica y fertilidad en los animales afectados. El diagnóstico temprano permitirá reducir pérdidas económicas por días abiertos.

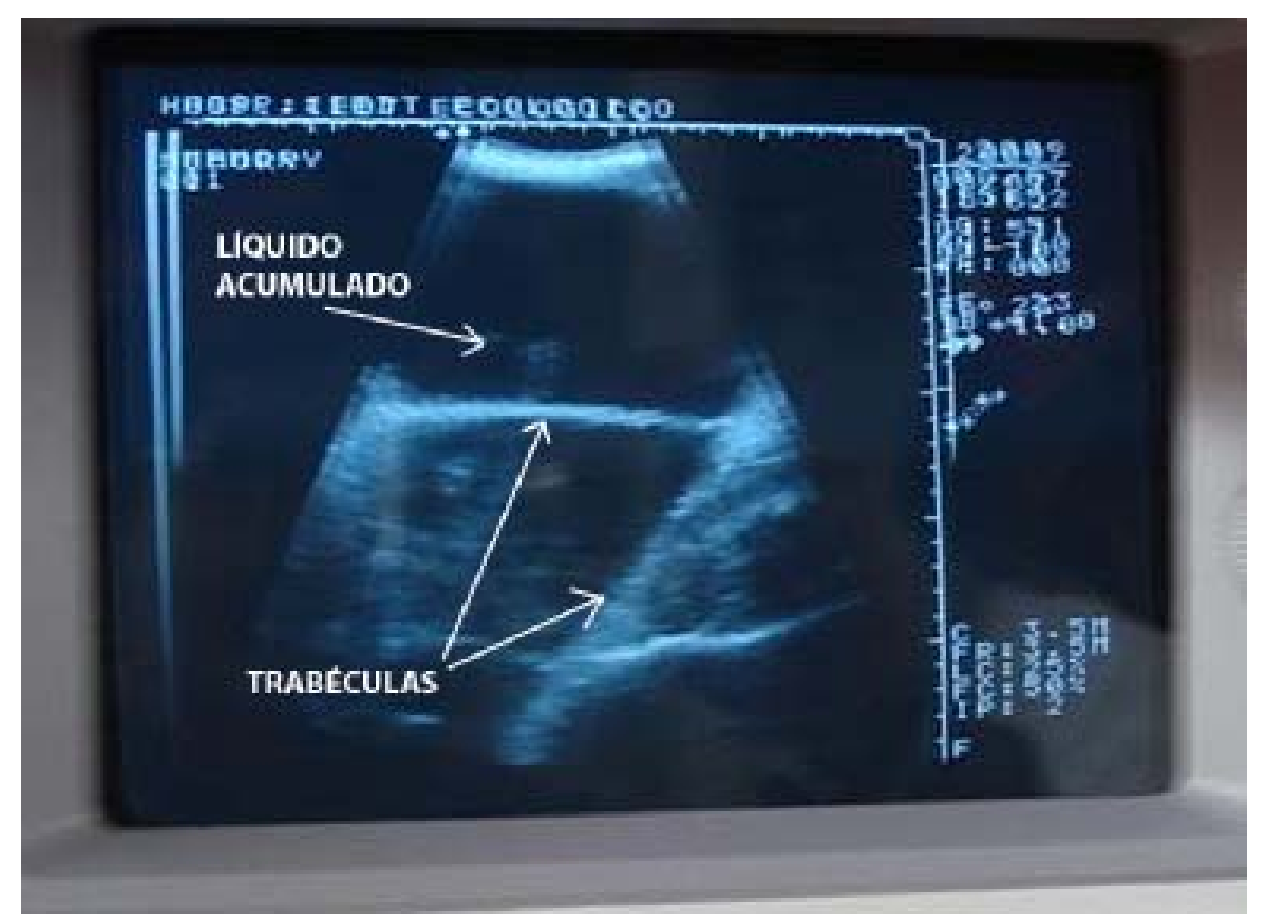

Figura 1. Ecografía de útero de cabra con pseudopreñez (presencia de trabéculas) 


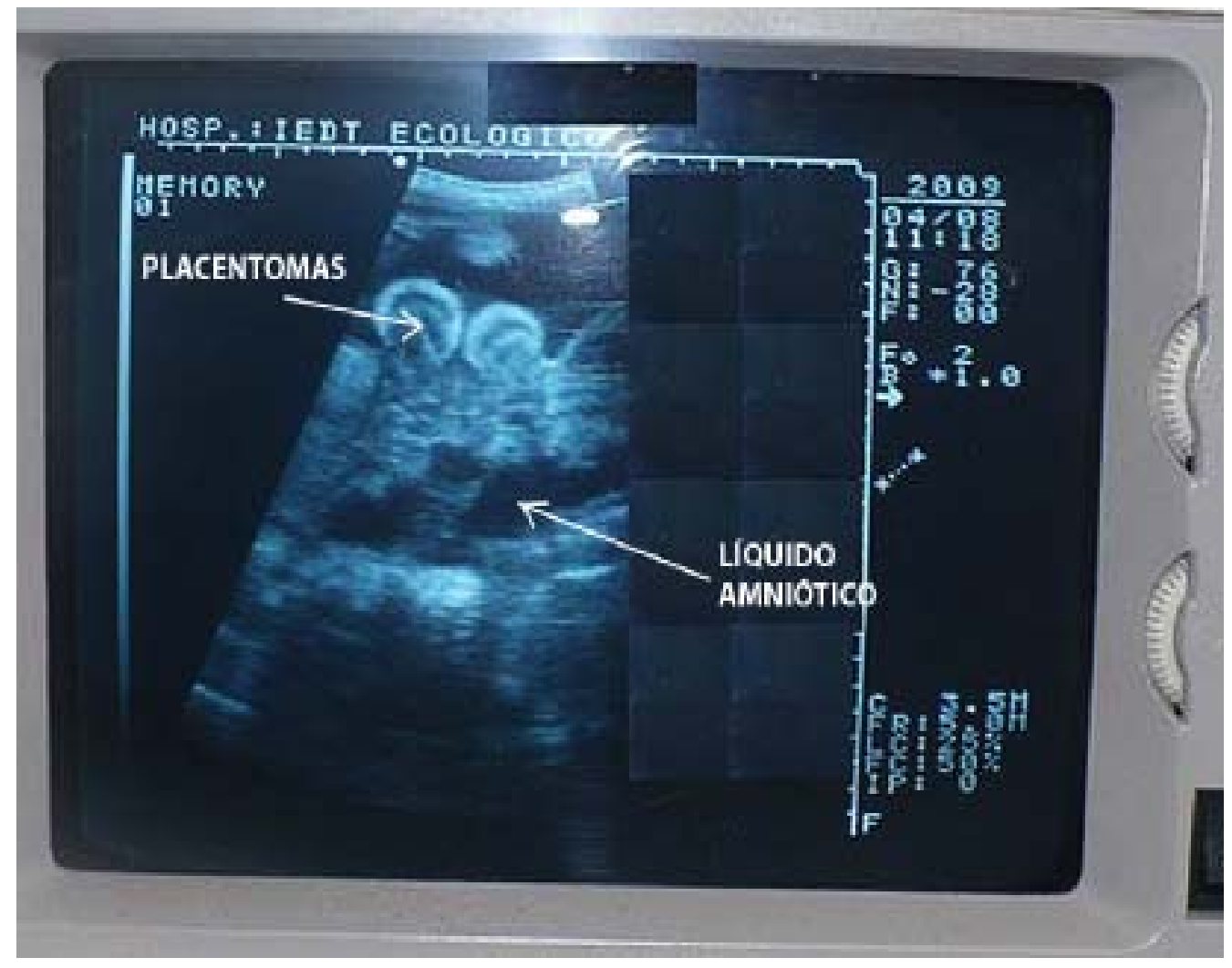

Figura 2. Ecografía de útero de cabra con gestación normal (presencia de placentomas)

\section{LITERATURA CITADA}

1. Arroyo O. 1998. Producción de caprinos. Lima: Ed Procabra. 33 p.

2. Batista M, Medina $P$, Calero $P$, González F, Quesada E, Gracia A. 2001. Incidence and treatment of hydrometra in Canary Island goats. Vet Rec 149: 329-330.

3. Hesselink JW. 1993. Incidence of hydrometra in dairy goats. Vet Rec 132: 110-112.
4. Madrid-Bury N, González-Stagnaro C. 1995. Pseudopreñez en cabras criollas venezolanas. Rev Científica FCVLUZ 5(2): 95-99.

5. Pieterse MC, Taverne MAM. 1986. Hydrometra in goats: Diagnosis with realtime ultrasound and treatment with prostaglandins or oxitocin. Theriogenology 26: 813-821.

6. Salles M, Araujo A. 2008. Pseudogestação em cabras leiteiras relato de caso. Vet Zootec 15: 251-256. 